INPLASY

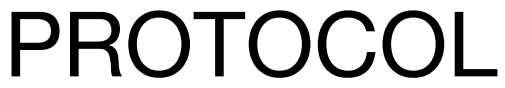

To cite: $\mathrm{Di}$ et al. Critical appraisal of the reporting quality of risk prediction models for idiopathic pulmonary fibrosis. Inplasy protocol 2020110105. doi: 10.37766/inplasy2020.11.0105

Received: 24 November 2020

Published: 24 November 2020

Corresponding author: Xueqing Yu

yxqshi@163.com

Author Affiliation: The First Affiliated Hospital of Henan University of Chinese Medicine

Support: NNSF(No.81973779).

Review Stage at time of this submission: Piloting of the study selection process.

Conflicts of interest: No conflict of interest.

\section{Critical appraisal of the reporting quality of risk prediction models for idiopathic pulmonary fibrosis}

\author{
Di, JQ1; Li, XL2; Yang, JJ3; Li, LG4; Yu, XQ55.
}

Review question / Objective: Since the development of risk prediction models, many more prediction models for IPF have been built with more complex statistical learning algorithms have been published. The Transparent Reporting of a multivariable prediction model for Individual Prognosis Or Diagnosis (TRIPOD) statement was published in 2015 in response to the rapid growth of clinical prediction models as well as the incomplete reporting of model development an validation studies. The aim of the study is to evaluate the quality of risk prediction models for idiopathic pulmonary fibrosis.

Condition being studied: We have developed a clear implementation procedure. First, we will systematically search electronic database to search risk models for IPF, and reference of all included article were assessed for relevant articles to ensure the comprehensiveness of the search; Second, we carried out basic data extraction of the included studies. Third, the TRIPOD statement was applied to analysis the reporting quality of IPF production models

INPLASY registration number: This protocol was registered with the International Platform of Registered Systematic Review and Meta-Analysis Protocols (INPLASY) on 24 November 2020 and was last updated on 24 November 2020 (registration number INPLASY2020110105).

\section{INTRODUCTION}

Review question / Objective: Since the development of risk prediction models, many more prediction models for IPF have been built with more complex statistical learning algorithms have been published. The Transparent Reporting of a multivariable prediction model for Individual Prognosis Or Diagnosis (TRIPOD) statement was published in 2015 in response to the rapid growth of clinical 
prediction models as well as the incomplete reporting of model development an validation studies. The aim of the study is to evaluate the quality of risk prediction models for idiopathic pulmonary fibrosis.

Condition being studied: We have developed a clear implementation procedure. First, we will systematically search electronic database to search risk models for IPF, and reference of all included article were assessed for relevant articles to ensure the comprehensiveness of the search; Second, we carried out basic data extraction of the included studies. Third, the TRIPOD statement was applied to analysis the reporting quality of IPF production models.

\section{METHODS}

Search strategy: We performed a systematic literature search of PubMed, Embase, and Web of Science from January 1, 2015 to November 2, 2020 to search risk models for IPF. The database search strategy combined the following terms: Nomograms, ROC Curve, Clinical prediction, Prognostic score, Net reclassification index, Calibration; Idiopathic pulmonary fibrosis, Pulmonary fibroses, idiopathic.

Participant or population: Patients with idiopathic pulmonary fibrosis.

Intervention: None.

Comparator: None.

Study designs to be included: The diagnosis or prognostic multivariable prediction models of idiopathic pulmonary fibrosis.

Eligibility criteria: Studies should predict diagnosis or prognosis of idiopathic pulmonary fibrosis.

Information sources: PubMed, Embase, and Web of Science from January 1, 2015 to November 2, 2020.
Main outcome(s): The report quality of risk prediction models of IPF.

Data management: Using Microsoft Excel to manage data.

Quality assessment / Risk of bias analysis: Assessment the report quality of idiopathic pulmonary fibrosis models based on Transparent Reporting of a multivariable prediction model for Individual Prognosis or Diagnosis (TRIPOD) statement.

Strategy of data synthesis: Calculate the Kappa value of internal consistency with SPSS.

Subgroup analysis: None.

Sensibility analysis: None.

Language: English.

Country(ies) involved: China.

Keywords: TRIPOD; Prediction model; Idiopathic pulmonary fibrosis; Development; Validation.

Contributions of each author:

Author 1 - Jiaqi Di.

Author 2 - Xuanlin Li.

Author 3 - Jingjing Yang.

Author 4 - Luguang Li.

Author 5 - Xueqing Yu. 